\title{
What do we know about obsessive-compulsive disorder following traumatic brain injury?
}

\author{
Michelle Rydon-Grange, ${ }^{\prime}$ and Rudi Coetzer ${ }^{2,3 *}$
}

\footnotetext{
${ }^{1}$ North Wales Clinical Psychology Programme, Bangor University, Bangor, Gwynedd, Wales, UK

${ }^{2}$ North Wales Brain Injury Service, Betsi Cadwaladr University Health Board NHS, Colwyn Bay, Wales, UK

${ }^{3}$ Centre for Rehabilitation, Oxford Brookes University, Oxford, UK
}

In addition to the well-known cognitive impairment following traumatic brain injury (TBI), neuropsychiatric sequelae are often reported as well. Although not the most common neuropsychiatric consequence of TBI, obsessive-compulsive disorder (OCD) has been associated with TBI. However, diagnosing new onset OCD secondary to TBI is complicated by the potential for cognitive impairment secondary to TBI masquerading as OCD. In particular, memory difficulties and executive dysfunction may be confused as representing obsessions and compulsions. Research in this area, which could guide clinical practice, remains limited. In addition to using Diagnostic and Statistical Manual of Mental Disorders, Fifth Edition (DSM-V) criteria, neuropsychological testing and collateral interviews may help clinicians when considering differential diagnoses in this complex area of neuropsychiatry.

Received 12 June 2015; Accepted 1 July 2015; First published online 28 August 2015

Key words: Cognitive impairment, DSM-V, neuropsychiatric disorder, obsessive-compulsive disorder, traumatic brain injury.

Traumatic brain injury (TBI) is a significant cause of disability. Impairments in physical, cognitive, psychological, and behavioral functioning are common. Neuropsychiatric disorders ${ }^{1-3}$ are also common sequelae of TBI. Posttraumatic psychiatric disorders are associated with poorer longer-term outcome ${ }^{4}$ and may hinder rehabilitation. Although not the most prevalent clinical presentation post-TBI, obsessive-compulsive symptoms have been reported after TBI. ${ }^{5-14,16}$ However, the relationship between TBI and obsessive-compulsive phenomena remains poorly understood. What is currently known about this complex area of neuropsychiatry?

Neuroanatomical models of OCD in patients without TBI implicate frontostriatal pathophysiology. ${ }^{17,18}$ The neuropsychology of idiopathic OCD has been extensively researched, with some conflicting results. Nevertheless, cognitive deficits have been reported, including executive and memory problems among others. A recent metaanalysis of neuropsychological studies in adults with OCD reported the magnitude of impairments to be in the medium range for executive functions (ie, planning,

*Address for correspondence: Dr. Rudi Coezter, Consultant Neuropsychologist, North Wales Brain Injury Service, Betsi Cadwaladr University Health Board, Colwyn Bay Hospital, Hesketh Road, Colwyn Bay, LL29 8AY, UK.

(Email: Rudi.Coetzer@wales.nhs.uk) response inhibition, cognitive flexibility), processing speed, and sustained attention, while small effect sizes were reported for visuospatial abilities and working memory. ${ }^{19}$

Prevalence estimates vary, with up to $14 \%^{20}$ of individuals with TBI reporting new-onset OCD. However, it is likely that factors such as sampling biases, the use of different instruments (eg, self-report versus structured clinical interview) to assess OCD symptomatology, and underreporting of symptoms may account for relatively large discrepancies in prevalence rates. Generally, the clinical features of post-TBI OCD are thought to be similar to those observed in idiopathic OCD. ${ }^{13}$ Furthermore, these clinical features seem to co-occur in the context of specific cognitive deficits, including impairments on tasks of executive function, memory, and general intellectual ability. However, some research has failed to find support for an overlap of executive deficits in an idiopathic OCD versus TBI-only groups. ${ }^{15}$ Nevertheless a commensurate pattern of clinical and neuropsychological features was reported by Berthier $e t a l^{11}$ in a sample of $10 \mathrm{TBI}$ patients who also fulfilled diagnostic criteria for OCD.

A few case reports of OCD after TBI also exist, ${ }^{7,10,12}$ including, for example, the interesting case of a male with contamination concerns and ritualistic behavior 
(eg, counting, motor compulsions, praying) to alleviate anxiety associated with obsessions. ${ }^{6}$ Not much is known about the temporal relationship between TBI and OCD. Obsessions and compulsions have been reported to emerge during the very early post-acute phase ${ }^{12,16}$ (ie, 1 week-2 months post-TBI), as well as up to 2 years post-injury. ${ }^{10}$

Cognitive impairments, such as deficits in memory, executive function, attention, and processing speed, are commonly observed in patients with OCD post-TBI,, , 7,13 which suggests a possible association between cognitive impairment and OCD secondary to TBI. Conversely, whether the presence of an anxiety disorder, such as OCD, influences specific aspects of cognitive functioning in TBI populations is unknown. Indeed, we know that a number of psychiatric disorders, including OCD, have reasonably well-established cognitive correlates (eg, attentional deficits associated with depression) in the wider population.

Current thinking suggests multiple potential etiologies in the development of post-TBI OCD. These include pre-existing variables (eg, coping style, premorbid personality), type and location of lesion (eg, orbitofrontal region and frontal-subcortical circuits), and psychological response to TBI-related disturbances (eg, cognitive deficits) and subsequent functional impairments. ${ }^{21}$ Nevertheless, numerous studies have linked damage to frontal, temporal, and cingulate regions, and the basal ganglia, or both, in patients with OCD secondary to TBI. ${ }^{8,9}$ However, the exact biological basis for OCD post-TBI remains poorly understood.

Post-injury psychological response to cognitive disability has also been posited to underpin the development and maintenance of anxiety disorders in this population, with some recent findings supporting this theory. ${ }^{2,22}$ Thus, cognitive deficits observed in participants with post-injury OCD may be due to the effects of general comorbidity on cognition, as opposed to OCD per se. Indeed, studies suggest that comorbidity is the rule rather than exception in TBI populations. ${ }^{2,24}$ Specific environmental (eg, substance use, preexisting psychopathology, family support) or biological (eg, additional organic illness) vulnerability factors are also likely to interact with injury-related variables to affect the experience and outcome of TBI.

Diagnosing OCD in the context of TBI is complicated due to the potential for overlapping symptomatology between conditions. ${ }^{7,8}$ Cognitive impairments associated with TBI clearly have the potential to mimic aspects of the clinical presentation of idiopathic OCD. For example, memory deficits following TBI may result in excessive checking of tasks, mirroring compulsions observed in OCD. Likewise, executive function impairments resulting in perseverative behavior may mimic the repetitive behavioral patterns typical of OCD.
Similarly, poorer speed of information processing following TBI may be indistinguishable from obsessional slowness. Thus, disentangling whether particular symptoms better reflect the well-known cognitive impairments characteristic of TBI, or new-onset OCD, is challenging. Memory performance may be a more useful cognitive indicator to consider when diagnosing OCD secondary to TBI, and a diagnosis of OCD secondary to TBI should probably be questioned when OCD-type symptomatology occurs alongside significant deficits in memory. ${ }^{7}$

The fifth edition of the Diagnostic and Statistical Manual of Mental Disorders ${ }^{23}$ outlines guidance for the diagnosis of neuropsychiatric comorbidities in TBI populations. According to DSM-V ${ }^{23}$ criteria, OCD symptomatology occurring secondary to a medical condition should be coded as "OCD due to Another Medical Condition." Furthermore, it is possible to specify whether OCD-like symptoms, appearance preoccupations, hoarding symptoms, hair-pulling, or skin-picking symptoms predominate the clinical picture. As highlighted above, there is limited research evidence supporting a direct pathophysiological link between damage to specific brain structures and pathways following TBI, and subsequent OCD. Thus, medical indicators (eg, functional and structural imaging techniques) may currently offer only modest utility in aiding diagnostic accuracy of OCD secondary to TBI. Accordingly, consideration of other factors not currently outlined in DSM-V ${ }^{23}$ may help to further confirm or reject a diagnosis of OCD post-injury. For example, the fact that cognitive impairment commonly observed early post-injury has the potential to mirror OCD symptoms requires careful consideration.

Structured neuropsychological testing may help elucidate whether specific behaviors typically associated with OCD (ie, excessive checking, compulsive and inflexible behavioral patterns) might better represent underlying cognitive impairment (eg, memory, executive deficits) commonly observed following TBI. Moreover, as time since injury elapses and the temporal link between injury and OCD onset becomes more ambiguous, the potential impact of nonbiological factors upon clinical presentation should be considered. For example, environmental changes (eg, change in living situation, disruption to established routines) or an increase in environmental demands (eg, return to employment) may exacerbate post-injury memory difficulties and accompanying anxiety. Such environmental changes may lead to excessive checking, repetitive behaviors, and associated anxiety, potentially being mistaken for new-onset OCD. Indeed, anxiety post-TBI may be a psychological response to cognitive disability (eg, memory difficulties) and the associated impairment in adaptive functioning, and not necessarily indicate the presence of "pure" OCD 
symptomatology. If OCD-like symptoms (eg, checking) after TBI are considered to actually reflect injury-related cognitive impairment (eg, memory), a diagnosis of "Major or Mild Neurocognitive Disorder due to TBI" should be considered. ${ }^{23}$

Despite much research in the area, in reality little is known about OCD secondary to TBI. Accurate prevalence data regarding OCD after TBI are lacking, as is our current understanding regarding key etiological mechanisms involved in the emergence of the disorder. The clinical phenomenology and cognitive functioning of TBI patients who report new-onset OCD are almost identical to idiopathic OCD samples, suggesting a possible association between both conditions, although the nature of this association remains unclear. DSM-V ${ }^{23}$ provides helpful guidance on diagnosing OCD in the context of a medical condition, but the potential for overlapping symptomatology between TBI and OCD makes reaching a diagnosis challenging. Ultimately, what is the relevance of what may appear to be an overly meticulous focus on diagnostic issues in a complex, multifactorial area of neuropsychiatric presentation? Accurate diagnostic formulation is the cornerstone of treatment plans. This is of course the case in brain injury rehabilitation also. "OCD after TBI," which in fact represents cognitive impairment, may respond better to cognitive rehabilitation (including memory compensatory strategies), whereas "true OCD" associated with TBI, may require different treatment strategies, including pharmacological and psychological (for example, cognitive behavior therapy and response prevention) approaches.

\section{Disclosures}

The authors do not have any disclosures, and the authors do not have any affiliation with or financial interest in any organization that might pose a conflict of interest.

\section{REFERENCES:}

1. Ciurli P, Formisano R, Bivona U, Cantagallo A, Angelelli P. Neuropsychiatric disorders in persons with severe traumatic brain injury: prevalence, phenomenology, and relationship with demographic, clinical, and functional features. J Head Trauma Rehabil. 2011; 26(2): 116-126.

2. Whelan-Goodinson R, Ponsford J, Johnston L, Grant F. Psychiatric disorders following traumatic brain injury: their nature and frequency. J Head Trauma Rehabil. 2009; 24(5): 324-332.

3. Koponen S, Taiminen T, Hiekkanen H, Tenovuo O. Axis I and II psychiatric disorders in patients with traumatic brain injury: a 12-month follow-up study. Brain Inj. 2011; 25(11): 1029-1034.

4. Diaz AP, Schwarzbold ML, Thais ME, et al. Psychiatric disorders and health-related quality of life after severe traumatic brain injury: a prospective study. J Neurotrauma. 2012; 29(6): 1029-1037.
5. Gould KR, Ponsford JL, Spitz G. Association between cognitive impairments and anxiety disorders following traumatic brain injury. J Clin Exp Neuropsychol. 2014; 36(1): 1-14.

6. Hofer H, Frigerio S, Frischknecht E, Gassmann D, Gutbrod K, Müri RM. Diagnosis and treatment of an obsessive-compulsive disorder following traumatic brain injury: a single case and review of the literature. Neurocase. 2013; 19(4): 390-400.

7. Coetzer BR. Does memory impairment exclude a diagnosis of OCD after traumatic brain injury? J Neuropsychiatry Clin Neurosci. 2011; 23(3): E12.

8. Coetzer BR. Obsessive-compulsive disorder following brain injury: a review. Int J Psychiatry Med. 2004; 34(4): 363-377.

9. Grados MA. Obsessive-compulsive disorder after traumatic brain injury. Int Rev Psychiatry. 2003; 15(4): 350-358.

10. Williams WH, Evans JJ, Fleminger S. Neurorehabilitation and cognitive-behaviour therapy of anxiety disorders after brain injury: an overview and case illustration of obsessive-compulsive disorder. Neuropsychol Rehabil. 2003; 13(1-2): 133-148.

11. Berthier ML, Kulisevsky J, Gironell A, López OL. Obsessivecompulsive disorder and traumatic brain injury: behavioral, cognitive, and neuroimaging findings. Neuropsychiatry Neuropsychol Behav Neurol. 2001; 14(1): 23-31.

12. Childers MK, Holland D, Ryan MG, Rupright J. Case study: Obsessional disorders during recovery from severe head injury: report of four cases. Brain Inj. 1998; 12(7): 613-616.

13. Berthier ML, Kulisevsky J, Gironell A, Heras JA. Obsessivecompulsive disorder associated with brain lesions: clinical phenomenology, cognitive function, and anatomic correlates. Neurology. 1996; 47(2): 353-361.

14. Kant R, Smith-Seemiller L, Duffy JJ. Obsessive-compulsive disorder after closed head injury: review of literature and report of four cases. Brain Inj. 1996; 10(1): 55-63.

15. Coetzer R, Stein DJ, Du Toit PL. Executive function in traumatic brain injury and obsessive-compulsive disorder: an overlap? Psychiatry Clin Neurosci. 2001; 55(2): 83-87.

16. Mallya S, Sutherland J, Pongracic S, Mainland B, Ornstein TJ. The manifestation of anxiety disorders after traumatic brain injury: a review. J Neurotrauma. 2015; 32(7): 411-421.

17. Melloni M, Urbistondo C, Sedeño L, Gelormini C, Kichic R, Ibanez A. The extended fronto-striatal model of obsessive compulsive disorder: convergence from event-related potentials, neuropsychology and neuroimaging. Front Hum Neurosci. 2012; 6: 259.

18. Rasmussen SA, Eisen JL, Greenberg BD. Toward a neuroanatomy of obsessive-compulsive disorder revisited. Biol Psychiatry. 2013; 73(4): 298-299.

19. Abramovitch A, Abramowitz JS, Mittelman A. The neuropsychology of adult obsessive-compulsive disorder: a meta-analysis. Clin Psychol Rev. 2013; 33(8): 1163-1171.

20. Hibbard MR, Uysal S, Kepler K, Bogdany J, Silver J. Axis I psychopathology in individuals with traumatic brain injury. J Head Trauma Rehabil. 1998; 13(4): 24-39.

21. Arlinghaus KA, Shoaib AM, Price TRP. Neuropsychiatric assessment. In Silver JM, McAllister TW, Yudofsky SC, eds. Textbook of Traumatic Injury. Washington, DC: American Psychiatric Publishing; 2005: 59-78.

22. Willmott C, Ponsford J, Hocking C, Schönberger M. Factors contributing to attentional impairments after traumatic brain injury. Neuropsychology. 2009; 23(4): 424-432.

23. American Psychiatric Association DSM-5 Task Force. Diagnostic and Statistical Manual of Mental Disorders, 5th ed. Washington, DC: American Psychiatric Association; 2013.

24. Rogers JM, Read CA. Psychiatric comorbidity following traumatic brain injury. Brain Inj. 2007; 21(13-14): 1321-1333. 